\title{
EXTREME POINTS OF THE UNIT BALL OF THE SPACE OF LIPSHITZ FUNCTIONS
}

\author{
JEFF D. FARMER
}

(Communicated by Dale Alspach)

\begin{abstract}
We consider the space of all Lipschitz functions on a metric space with bounded Lipschitz norm, and give an intrinsic characterization of the extreme points of the unit ball. We briefly discuss some examples of extreme Lipschitz functions, and apply the result to show that if the norm of a Banach space is Gateaux differentiable then extreme functions on any one-dimensional subspace may be canonically extended to extreme functions on the whole space.
\end{abstract}

Let $(X, d)$ be a metric space. The most natural real functions to consider on $(X, d)$ are the Lipschitz functions, that is, the functions which increase distances in a bounded way. If $f$ is a real valued function on $X$, then we define the Lipschitz "norm" of $f$ as follows:

$$
\|f\|_{\text {Lip }}=\sup _{x, y \in X}\left\{\frac{|f(x)-f(y)|}{d(x, y)}\right\} .
$$

This supremum defines a seminorm on the vector space of real functions on which it exists for any metric space $X$; it will distinguish between any two functions whose difference is not constant. The most common way to actually obtain a norm on this vector space is to take the maximum of the Lipschitz norm given above with the supremum of the function $f$ on the space. To do this one must either consider only bounded $f$ or require the underlying metric space to be compact. An advantage of this approach is that the Banach space of Lipschitz functions obtained is actually a Banach algebra. This space of functions and other closely related spaces have been the subject of considerable study (see, for example, [Ro, Jo, Ma, S, dL]). Information has been obtained about the dual space of the space of Lipschitz functions, extreme points of the unit ball, isometries of the spaces and the exact nature of the space of Lipschitz functions on certain classical objects (some Banach spaces, the unit interval, etc.).

There is another way to obtain a norm on the vector space of Lipschitz functions, and that is to identify a distinguished point of the metric space and

Received by the editors October 12, 1992.

1991 Mathematics Subject Classification. Primary 46B20.

Key words and phrases. Lipschitz functions, extreme points.

This paper forms a portion of the author's Ph.D. dissertation under the supervision of W. B. Johnson. 
consider only the functions which are zero at this point. This Banach space is sometimes denoted by $\operatorname{Lip}_{0}(X, d)$, but we will call it $X^{\#}$ following Lindenstrauss [Li], who considered the spaces formed when the underlying metric space is a Banach space. In this context, we see that this method of norming the Lipschitz functions is the most natural one, since any vector space has a natural distinguished point, namely 0 . We will call $X^{\#}$ the Lipschitz dual of $X$, where $X$ is any metric space with a distinguished point 0 .

We first observe that we do not lose any information by choosing a distinguished point; indeed, any two versions of $X^{\#}$ we form are isometric (simply add the difference between the value of each function at the old zero and the value at the new zero to each function). We will always denote the distinguished point of a metric space $X$ by 0 . We can also note that the metric space $X$ embeds isometrically into the Banach space dual of $X^{\#}$ by taking each point to the functional which evaluates a function in $X^{\#}$ at that point. There are various functional analytic questions which can be asked about these basic relationships and their implications. One of the most basic is a consideration of the extreme points of the unit ball of $X^{\#}$.

Rolewicz [Rol2] has characterized the extreme points of the unit ball of $[0,1]^{\#}$ as being those functions whose derivative is one almost everywhere, and has shown in [Rol1] that a similar theorem cannot hold for $([0,1] \times[0,1])^{\#}$ (in the Euclidean metric). Gobszas [G] has given some conditions which are either necessary or sufficient for functions to be extreme in the general case and has characterized extreme points for a restricted class of metric spaces. Below (in an independent result) we completely characterize the extreme functions, and apply the result by showing when some very simple functions (formed using linear functionals) can be extreme.

We begin with the characterization of extreme functions.

Theorem 1. Let $X$ be a metric space. Then for $f$ in the unit ball we have

$$
f \in \operatorname{ext} B_{X^{*}} \Leftrightarrow \varepsilon_{x, y}^{f}=0 \quad \forall x, y \in X \quad\left(\Leftrightarrow \varepsilon_{x, 0}^{f}=0 \forall x \in X\right)
$$

where

$$
\begin{aligned}
\varepsilon_{x, y}^{f}=\inf \{\varepsilon \mid \exists & \left\{x=x_{0}, x_{1}, \ldots, x_{n}=y\right\} \\
& \left.\subset X \ni d\left(x_{i-1}, x_{i}\right)-\varepsilon_{i} \leq\left|f\left(x_{i-1}\right)-f\left(x_{i}\right)\right|\right\}
\end{aligned}
$$

with $\varepsilon_{i}>0$ and $\left.\sum_{i=1}^{n} \varepsilon_{i} \leq \varepsilon\right)$.

Proof. The parenthetical equivalence follows obviously from the observation that $\varepsilon_{x, y}^{f}+\varepsilon_{y, z}^{f} \geq \varepsilon_{x, z}^{f}$ for all $x, y, z \in X$.

Now suppose first that $\varepsilon_{x, 0}^{f}=0 \forall x$ and let $f=(g+h) / 2$, with $f, g, h \in$ $B_{X^{*}}$. We show that $f \equiv g \equiv h$. So let $\delta>0$ and get $0=x_{0}, x_{1}, \ldots, x_{n}=x$ such that

$$
d\left(x_{i-1}, x_{i}\right)-\varepsilon_{i} \leq\left|f\left(x_{i-1}\right)-f\left(x_{i}\right)\right| \leq d\left(x_{i-1}, x_{i}\right) \text { and } \sum_{i=1}^{n} \varepsilon_{i} \leq \delta .
$$

We will describe this situation by saying that $0=x_{0}, x_{1}, \ldots, x_{n}=x$ is a chain which $\delta$-connects 0 and $x$. We begin with $f\left(x_{1}\right)$, supposing without loss of generality that $f\left(x_{1}\right)>0$ and that $f\left(x_{1}\right)-g\left(x_{1}\right)=h\left(x_{1}\right)-f\left(x_{1}\right) \geq 0$ (the other cases are exactly similar). But since $h$ is also in the unit ball we combine

$$
f\left(x_{1}\right) \geq d\left(x_{1}, x_{0}\right)-\varepsilon_{1} \text { and } h\left(x_{1}\right) \leq d\left(x_{1}, x_{0}\right)
$$


to obtain $f\left(x_{1}\right)-g\left(x_{1}\right)=h\left(x_{1}\right)-f\left(x_{1}\right) \leq \varepsilon_{1}$.

We observe first that in general if $x, y \in X$ and $\varepsilon>0$ such that $d(x, y)-$ $\varepsilon \leq|f(x)-f(y)|$ then we will have

$$
|(f(x)-f(y))-(g(x)-g(y))| \leq \varepsilon .
$$

Indeed,

$$
\begin{aligned}
-\varepsilon & =d(x, y)-\varepsilon-d(x, y) \leq(f(x)-f(y))-(g(x)-g(y)) \\
& =(h(x)-h(y))-(f(x)-f(y)) \leq d(x, y)-(d(x, y)-\varepsilon)=\varepsilon .
\end{aligned}
$$

A similar observation gives us the induction as follows. Suppose that we have established that

$$
\left|f\left(x_{k}\right)-h\left(x_{k}\right)\right|=\left|f\left(x_{k}\right)-g\left(x_{k}\right)\right| \leq \beta .
$$

Then by the observation we may write

$$
\begin{aligned}
\left|g\left(x_{k+1}\right)-f\left(x_{k+1}\right)\right| & =\left|g\left(x_{k+1}\right)-g\left(x_{k}\right)-\left(f\left(x_{k+1}\right)-f\left(x_{k}\right)\right)+g\left(x_{k}\right)-f\left(x_{k}\right)\right| \\
& \leq \varepsilon_{k}+\beta .
\end{aligned}
$$

Thus

$$
\left|f\left(x_{k+1}\right)-h\left(x_{k+1}\right)\right|=\left|f\left(x_{k+1}\right)-g\left(x_{k+1}\right)\right| \leq \varepsilon_{k}+\beta
$$

and by summing we obtain $|f(x)-h(x)|=|f(x)-g(x)| \leq \delta$. Since $\delta$ and $x$ were arbitrary, we conclude that $f$ is extreme and finish the first implication.

To prove the converse, consider $f$ a function in the unit ball and suppose that there exists $y \in X$ such that $\varepsilon_{y, 0}>0$. Let $Y$ be the set of all such $y \in X$ and notice that for each such $y$

$$
\varepsilon_{x, y}=\varepsilon_{y, 0} \quad \forall x \in X-Y .
$$

Define

$$
g(x)= \begin{cases}f(x)+\varepsilon_{x}, 0, & x \in Y, \\ f(x), & x \in X-Y,\end{cases}
$$

and

$$
h(x)= \begin{cases}f(x)-\varepsilon_{x}, 0, & x \in Y, \\ f(x), & x \in X-Y .\end{cases}
$$

Then $f=(g+h) / 2$. We must show that $|g(x)-g(y)| \leq d(x, y) \quad \forall x, y \in X$ and similarly for $h$. If neither $x$ nor $y$ is in $Y$, this is clear, so suppose first that $x \in X-Y$ and $y \in Y$. Then

$$
|f(x)-f(y)| \leq d(x, y)-\varepsilon_{x, y} \leq d(x, y)-\varepsilon_{y, 0} .
$$

So

$$
|g(x)-g(y)|=\left|f(x)-\left(f(y)+\varepsilon_{y}, 0\right)\right| \leq|f(x)-f(y)|+\varepsilon_{y, 0} \leq d(x, y) .
$$

In the case where both $x$ and $y$ are in $Y$, first observe that we always have $\varepsilon_{z, w}+\varepsilon_{w, u} \geq \varepsilon_{z, u}$. Then write

$$
\begin{aligned}
|g(x)-g(y)| & =\left|f(x)+\varepsilon_{x, 0}-f(y)-\varepsilon_{y, 0}\right| \leq|f(x)-f(y)|+\left|\varepsilon_{x, 0}-\varepsilon_{y, 0}\right| \\
& \leq d(x, y)-\varepsilon_{x, y}+\left|\varepsilon_{x, 0}-\varepsilon_{y, 0}\right| \leq d(x, y) .
\end{aligned}
$$

The same calculation clearly works for $h$. 
Given an extreme function $f$ and chain of elements $\left\{x_{i}\right\}_{i=1}^{n}$ connecting $x$ and $y$ to within $\varepsilon$ as in Theorem 1, we say that an element $x_{i}$ of the chain is positive if

$$
f\left(x_{i}\right)-f\left(x_{i+1}\right) \geq d\left(x_{i}, x_{i+1}\right)-\varepsilon_{i+1}
$$

and

and negative if

$$
f\left(x_{i}\right)-f\left(x_{i-1}\right) \geq d\left(x_{i}, x_{i-1}\right)-\varepsilon_{i},
$$

$$
f\left(x_{i+1}\right)-f\left(x_{i}\right) \geq d\left(x_{i}, x_{i+1}\right)-\varepsilon_{i+1}
$$

and

$$
f\left(x_{i-1}\right)-f\left(x_{i}\right) \geq d\left(x_{i}, x_{i-1}\right)-\varepsilon_{i} .
$$

Our first claim about these definitions is that any chain can be replaced by a chain where all of the elements are either positive or negative with no increase in $\varepsilon$. If an element is neither positive or negative, then one must have

$$
f\left(x_{i}\right)-f\left(x_{i+1}\right) \geq d\left(x_{i}, x_{i+1}\right)-\varepsilon_{i+1} \text { and } f\left(x_{i-1}\right)-f\left(x_{i}\right) \geq d\left(x_{i}, x_{i-1}\right)-\varepsilon_{i}
$$

or the same thing with the roles of $i+1$ and $i-1$ reversed. If this is the case, we can remove $x_{i}$ from the chain, obtaining by adding the above

$$
f\left(x_{i-1}\right)-f\left(x_{i+1}\right) \geq d\left(x_{i}, x_{i+1}\right)-\varepsilon_{i+1}-\varepsilon_{i} .
$$

Thus we obtain alternating positive and negative elements. We can now also assume that $d\left(x_{i}, x_{i-1}\right)-\varepsilon_{i}>0$ because if it is not for some index, we may remove both $x_{i}$ and $x_{i-1}$. To see this assume without loss of generality that $x_{i}$ is negative, and write

$$
\begin{aligned}
f\left(x_{i+1}\right)-f\left(x_{i-2}\right) & =f\left(x_{i+1}\right)-f\left(x_{i}\right)+f\left(x_{i}\right)-f\left(x_{i-1}\right)+f\left(x_{i-1}\right)-f\left(x_{i-2}\right) \\
& \geq f\left(x_{i+1}\right)-f\left(x_{i}\right)-d\left(x_{i}, x_{i-1}\right)+f\left(x_{i-1}\right)-f\left(x_{i-2}\right) \\
& \geq f\left(x_{i+1}\right)-f\left(x_{i}\right)-d\left(x_{i}, x_{i-1}\right)+f\left(x_{i-1}\right)-f\left(x_{i-2}\right) \\
& \geq d\left(x_{i+1}, x_{i}\right)-\varepsilon_{i+1}-d\left(x_{i}, x_{i-1}\right)+d\left(x_{i-1}, x_{i-2}\right)-\varepsilon_{i-1} \\
& \geq d\left(x_{i+1}, x_{i-2}\right)-2 d\left(x_{i}, x_{i-1}\right)-\varepsilon_{i+1}-\varepsilon_{i-1} \\
& \geq d\left(x_{i+1}, x_{i-2}\right)-2 \varepsilon_{i}-\varepsilon_{i+1}-\varepsilon_{i-1} .
\end{aligned}
$$

If the same relation holds with $x_{i-2}$ and $x_{i+1}$ we can delete them as well; by a calculation similar to that above we still at most double $\varepsilon$.

Whenever we are talking about a particular chain we will assume that we have cleaned it up as above and we will call the set of positive elements $P$ and the set of negative elements $N$.

Lemma 2. Let $E$ be a closed subset of a metric space $X$ (assume $E$ contains the 0$)$, and let $f$ be an extreme point of the unit ball of $E^{\#}$. Define

$$
f_{p}(x)=\sup _{y \in E}\{f(y)-d(x, y)\}, \quad f_{n}(x)=\inf _{y \in E}\{f(y)+d(x, y)\} .
$$

Then both $f_{p}$ and $f_{n}$ are extreme points in the unit ball of $X^{\#}$.

Proof. It is clear that there is an appropriate chain joining any two elements in $E$, and that any element in $X$ can be $\varepsilon$-connected to $E$ in one step. But by our criterion, this is enough.

Remark. There are obviously many possible extensions; one way of constructing them is to take an increasing chain of closed subsets and iterate the above 
process, alternating between the two kinds of extensions. In fact, in some sense, all extreme points of the ball look more or less like this (see Proposition 3).

Our next comment concerns the situation when $X$ is a finite set. In this case a compactness argument quickly shows that there must exist chains linking every pair of elements exactly. Therefore each extreme point is determined by a partition $X=P \cup N \cup M$ where each element of $M$ is a metric middle point of a pair of elements, one from $N$ and one from $P$, and a graph which connects every element of $P$ to at least one element of $N$. The set of such elements is finite and the space $X^{\#}$ is polyhedral. Since these extreme functions have a nice form it would be helpful to know whether we can use them to approximate any extreme function in an appropriate sense. We answer this question as follows.

Proposition 3. Let $D$ be a dense subset of a metric space $X$, and let

$$
F=\left\{f_{p}: f \in \operatorname{ext}\left(B_{E^{*}}\right), E \subset D,|E|<\infty\right\} .
$$

Then $F$ is weak star dense in the extreme points of the ball of $X^{\#}$, and hence the ball will be the weak star closed convex hull of $F$.

Proof. If we consider the space $X^{\#}$ with the topology of pointwise convergence from $X$, we see it has a linear dual which is the predual of $X^{\#}$; this is what we mean by the weak star topology on $X^{\#}$ (it is the same as the topology of pointwise convergence). With this remark, the proposition is clear.

We now wish to consider some extreme points in the ball of $Y^{\#}$ which are particularly simple, in the case where $Y$ is some subset of a Banach space $X$. Suppose $f \in[-1,1]^{\#}$ is an extreme point of the unit ball; by Rolewicz [Rol2], we know that it is a function with $\left|f^{\prime}\right|=1$ almost everywhere. Now consider a norm one linear functional $x^{*} \in X^{*}$; we can consider $f \circ x^{*}$, which is a Lipschitz function on $Y$. If $f$ is a norm one Lipschitz function on the real line, $f \circ x^{*}$ will be a Lipschitz function of norm one on all of $X$. We can ask whether functions formed in this manner are extreme points in the ball of $X^{\#}$. This question turns out to be related closely to whether or not the functional $x^{*}$ is extreme in $X^{*}$.

Theorem 4. Let $x$ be a point of norm Gateaux differentiability on the unit sphere of a real Banach space $X$, and let $f \in \mathscr{R}^{\#}$ be an extreme point of the unit ball. Then $f \circ x^{*}$ is an extreme point of the ball in $X^{\#}$, where $x^{*}$ is the unique norming functional for $x$.

Proof. It is obvious that the function has norm one. It suffices to show that for any $y \in X$ and any $\varepsilon_{0}>0$ there exists a chain $\varepsilon_{0}$-connecting $y$ to $x^{*}(y) x$ with respect to the function $f \circ x^{*}$, since all points in the one-dimensional subspace spanned by $x$ are connected to one another by the extremity of $f$. We can also assume that $f^{\prime}\left(x^{*}(y)\right)=1$ since points where $\left|f^{\prime}\left(x^{*}(y)\right)\right|=1$ are dense and the argument below is symmetric with respect to sign.

Choose $\delta<1$ to satisfy

$$
f\left(x^{*}(y)\right)-f\left(x^{*}(y)-\delta\right)>(1-\beta) \delta
$$

with $\beta$ to be specified later. Let $w=\delta x$ and $z=y-x^{*}(y) x$. Since the norm is Gateaux differentiable at $x$ (therefore also at $w$ ), we can choose $h$ small enough that

$$
(\|w+h z\|-\|w\|) / h<\eta
$$


and so that $1 / h$ is an integer (with $\eta$ to be specified in a moment). Now let

$$
y_{2 n-1}=y-n h z-w \text { and } y_{2 n}=y-n h z, \quad n=0,1,2, \ldots, 1 / h .
$$

Notice that $x^{*}\left(y_{2 n-1}\right)=x^{*}(y)-\delta$ and that $x^{*}\left(y_{2 n}\right)=x^{*}(y)$. Now calculate

$$
d\left(y_{2 n}, y_{2 n-1}\right)-\left|\left(f \circ x^{*}\right)\left(y_{2 n}\right)-\left(f \circ x^{*}\right)\left(y_{2 n-1}\right)\right| \leq \delta-(1-\beta) \delta
$$

and

$d\left(y_{2 n}, y_{2 n+1}\right)-\left|\left(f \circ x^{*}\right)\left(y_{2 n}\right)-\left(f \circ x^{*}\right)\left(y_{2 n+1}\right)\right|=\|w+h z\|-(1-\beta) \delta<h \eta+\beta \delta$.

Multiplying each of these bounds by $1 / h$ and adding we obtain a bound for the $\varepsilon$ associated with this chain

$$
\varepsilon=\sum_{n=1}^{2 / h} \varepsilon_{n} \leq\left(\frac{1}{h}\right)(\beta \delta)+\left(\frac{1}{h}\right)(h \eta+\beta \delta)=\left(\frac{2}{h}\right) \beta \delta+\eta
$$

and by specifying $\beta<\varepsilon_{0}(h / 4)$ and $\eta<\varepsilon_{0} / 2$ we obtain the desired chain.

On the other hand, if a functional is not extreme, then it is not the unique norming functional for any point. Lipschitz functions formed in the above manner using nonextreme points cannot be extreme.

Theorem 5. Let $\eta$ be a point of the unit sphere of $X^{*}$ which norms $x$ and which is not extreme; let $f \in R^{\#}$. Then $f \circ \eta$ is not an extreme point of the ball in $X^{\#}$.

Proof. If $f$ is not already an extreme point of the ball of $R^{\#}$, there is nothing to prove. Let $\eta=(\phi+\psi) / 2$. Note that $\phi$ and $\psi$ also norm $x$. Consider

$$
f \circ \eta=\frac{\{f \circ \eta+(\phi-\eta)\}+\{f \circ \eta+(\psi-\eta)\}}{2} .
$$

Write $X=\operatorname{ker} \eta \oplus[x]$ with $\|x\|=1$. Let $y=a x+v$ and $z=b x+w$ with $v, w \in \operatorname{ker} \eta$ and calculate

$$
\begin{aligned}
& |(f \circ \eta)(a x+v)+(\phi-\eta)(a x+v)-\{(f \circ \eta)(b x+w)+(\phi-\eta)(b x+w)\}| \\
& =|f(a)-f(b)+\phi(v)-\phi(w)| .
\end{aligned}
$$

Now assume without loss of generality that $f(a)-f(b)>0$ and that $a-b>0$ (other cases are symmetric), and recall that for $v, w \in \operatorname{ker} \eta$ we have $\phi(v)-$ $\phi(w)=\psi(w)-\psi(v)$. Letting $\theta$ stand for either $\phi$ or $\psi$ as needed, we can rewrite the above as

$$
\begin{aligned}
& |f(a)-f(b)+\phi(v)-\phi(w)|=f(a)-f(b)+\theta(v)-\theta(w) \\
& \quad \leq a-b+\theta(v)-\theta(w)=\theta((a x+v)-(b x+w)) \leq d((a x+v),(b x+w)) .
\end{aligned}
$$

A similar calculation shows that the other function is also in the unit ball. We thus see that $f \circ \eta$ is not extreme.

I would like to thank W. B. Johnson for several helpful comments.

\section{REFERENCES}

[dL] K. de Leeuw, Banach spaces of Lipschitz functions, Studia Math. 21 (1961), 55-66.

[G] S. Gobzas, Extreme points in Banach spaces of Lipschitz functions, Mathematica 31 (54) (1989), 25-33. 
[Jo] J. Johnson, Banach spaces of Lipschitz functions and vector-valued Lipschitz functions, Trans. Amer. Math. Soc. 148 (1970), 147-169.

[Li] J. Lindenstrauss, On nonlinear projections in Banach spaces, Michigan Math. J. 11 (1964), 263-287.

[Ma] E. Mayer-Wolf, Isometries between Banach spaces of Lipschitz functions, Israel J. Math. 38 (1981), 58-74.

[Rol1] S. Rolewicz, On extremal points of the unit ball in the Banach space of Lipschitz continuous functions, J. Austral. Math. Soc. Ser. A 41 (1986), 95-98.

[Rol2] __ On optimal observability of Lipschitz systems, Selected Topics in Operations Research and Mathematical Economics, Lecture Notes in Econom. and Math. Systems, vol. 226, Springer-Verlag, Berlin, 1984, pp. 151-158.

[Ro] A. K. Roy, Extreme points and linear isometries of the Banach space of Lipschitz functions, Canad. J. Math. 20 (1968), 1150-1164.

[S] D. Sherbert, The structure of ideals and point derivations in Banach algebras of Lipschitz functions, Trans. Amer. Math. Soc. 111 (1964), 240-272.

Department of Mathematics, University of Missouri, Columbia, Missouri 65211

Department of Mathematical Sciences, University of Northern Colorado, Greeley, COLORADO 80639

E-mail address: jfarmer@GoldnG8. univnorthco.edu 